\title{
Variation of median nerve and lateral antebrachial cutaneous nerve: One case report
}

\author{
Ze Jiang ${ }^{1}$, Lina Leng ${ }^{2}$, Daowen $\mathrm{Si}^{3 *}$ and Tiantao Wang ${ }^{3}$ \\ ${ }^{1}$ Anesthesia Class 1 Grade 2014, North China University of Science and Technology, No.57, Jianshenan Road, Tangshan063000, HebeiProvince, China \\ ${ }^{2}$ Clinical class 6 Grade 2013, North China University of Science and Technology, No.57, Jianshenan Road, Tangshan063000, HebeiProvince, China \\ ${ }^{3}$ Department of Anatomy of College of BasicMedical Sciences, North China University of Science and Technology, No.57, Jianshenan Road, Tangshan063000, \\ HebeiProvince, China
}

\begin{abstract}
During our routine dissection, we observed two variations in an old male cadaver. Firstly, the lateral root and the medial root that form the left median nerve descend in a longer distance than usual and converge into the median nerve trunk, and there is a communicating branch between the two roots. Secondly, the lateral antebrachial cutaneous nerve and the superficial branch of the radial nerve collectively dominate the area of the thumb-index web. This type of variations of the nerves is rare to see and should be considered to interpret atypical clinical presentations and avoid unusual injury during surgery.
\end{abstract}

\section{Case report}

Two anatomic variations are spotted during a dissection of peripheral nerves and vessels, which was conducted on an old male cadaver. The first anatomic variation is spotted at the left median nerve, where the lateral root and the medial root that form the left median nerve descend in a longer distance than usual and converge into the median nerve trunk. A communicating branch is also observed between the two roots. The second variation appears at the distribution of the lateral antebrachial cutaneous nerve, where the lateral antebrachial cutaneous nerve and the superficial branch of the radial nerve collectively dominate the area of the thumb-index web. The discovery of both variations is rare and reports on both at the same time have not been found. This report illustrates the two variations in details and explains the consequences they lead to. It also provides first hand anatomic data and great referential value for the future clinical work.

According to the first variation in this case, the two unusually long roots of the left median nerve, the lateral root and the medial root, are emitted from the fasciculus medialis plexus brachialis and the fasciculus lateralis plexus brachialis. According to the measurements during the dissection, the lengths of the two roots are $10.76 \mathrm{~cm}$ and $12.05 \mathrm{~cm}$, with diameters $2.44 \mathrm{~mm}$ and $2.87 \mathrm{~mm}$ respectively. As a result, the two axillary arteries descend and converge into the median nerve trunk in front of the brachial artery. The transverse diameter of the median nerve trunk is $3.66 \mathrm{~mm}$ after confluence. It goes down along the meidal bicipital groove with accompany of the brachial artery. In addition, a communicating branch is discovered between the lateral root and the medial root. The communicating branch starts from the medial root and terminates at a distance of $4.52 \mathrm{~cm}$ from the starting point of the lateral root. The length of the communicative branch is $4.28 \mathrm{~cm}$, with a transverse diameter of $1.12 \mathrm{~mm}$.

There is another variation of distribution of the lateral antebrachial cutaneous nerve in this case. The left musculocutaneous nerve passes through the deep fascia from the lateral biceps tendon and transits to the lateral antebrachial cutaneous nerve. The ateral antebrachial cutaneous nerve has a diameter of $2.20 \mathrm{~mm}$ and is distributed under the lateral skin of the forearm. After going down $23.48 \mathrm{~cm}$ in distance, the end of the lateral antebrachial cutaneous nerve is divided into 4 branches from the radial side to the ulnar side, having transverse diameters of $0.97 \mathrm{~mm}, 0.51 \mathrm{~mm}, 0.75 \mathrm{~mm}$ and $0.52 \mathrm{~mm}$ respectively. It then enters the thumb-index web and distributes under the skin of the area. Meanwhile, the radial nerve emits the superficial branch at the epicondylus lateralis humeri and then turns to the opisthenarin at the distal $1 / 3$ of the forearm. The transverse diameter of the superficial branch is $2.02 \mathrm{~mm}$. At a distance of $18.31 \mathrm{~cm}$ from the starting point, the superficial branch is divided into 2 sub branches, with transverse diameter $1.17 \mathrm{~mm}, 1.27 \mathrm{~mm}$ respectively. In the end it enters the thumbindex web. The superficial branch of the radial nerve and the lateral antebrachial cutaneous nerve form a communicating branch at the distal end of the radial styloid process. The communicating branch subsequently joins in the opisthenarin and is distributed under the skin of the thumb-index web.

There are two referential uses that can be extracted from these two variations. Firstly, according to the relevant information about the region of median nerve, the lateral and medial roots of median nerve are $2.77 \mathrm{~cm}$ and $2.83 \mathrm{~cm}$ long on average. Moreover, they are more merged into the median nerve at the axilla and are less located in the

Correspondence to: Daowen $\mathrm{Si}$, Department of Anatomy of College of BasicMedical Sciences, North China University of Science and Technology, No.57, Jianshenan Road, Tangshan063000, HebeiProvince, China, E-mail: sidaowen@126.com, Tel: 8603158805538

Key words: Hemoglobin and Methemoglobin catabolism, brain capillary, ferric iron deposition, blood brain barrier, apoptosis, neurovascular unit, neurodegenerative brain disease.

Received: September 20, 2017; Accepted: October 11, 2017; Published: October 13, 2017 
arm, which only accounts for $(9.7 \pm 1.6) \%$. In this case, however, the lateral and medial roots of the left median nerve descend in a longer distance and converge into the median nerve in the middle of the arm, which occurs rarely. Additionally, the communicative branch between the lateral and medial roots is also unusual. Therefore, the variation of the lateral and the medial roots of the left median nerve can be referred to in performing arm related surgery [1].

Secondly, the superficial radial nerve mainly dominates the thumb-index web. However in this case, the terminal branch of the lateral antebrachial cutaneous nerve and the superficial branch of the radial nerve conjointly form the communicative branch in the thumbindex web, which innervate the skin feeling of this region collectively. Therefore, the possibility of having this variation should be taken into

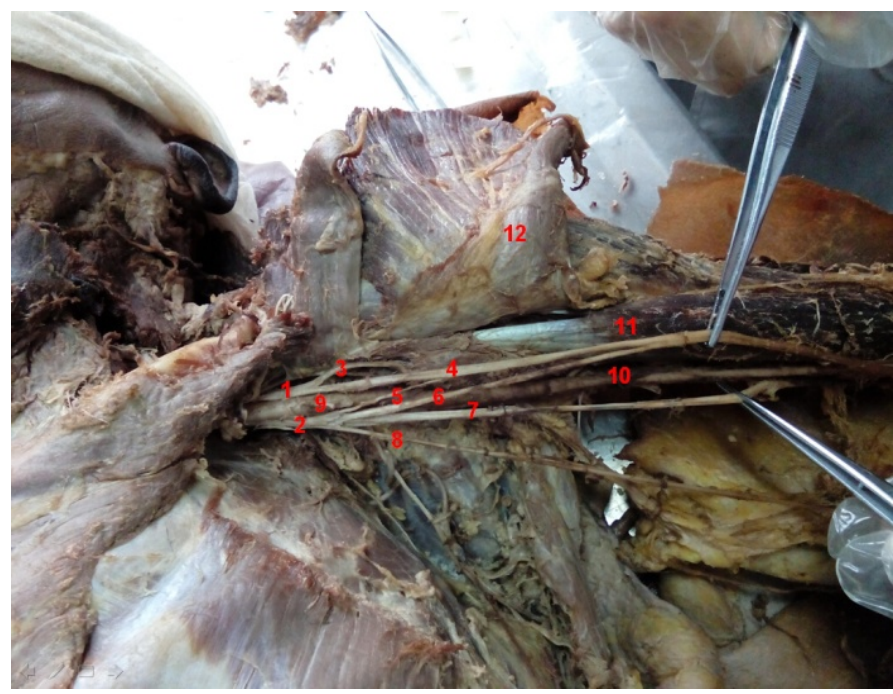

Figure 1. Variation of the median nerve origin

1. Fasciculus lateralis plexus brachialis, 2. Fasciculus medialis plexus brachialis, 3 . musculocutaneous nerve, 4. Lateral root of median, 5. Communicating branch between Lateral and medial roots of median nerve, 6. Medial root of median nerve, 7. Ulnar nerve, 8. Medial antebrachialcutaneous nerve, 9. Axillary artery, 10. Brachial artery, 11. Biceps brachii, 12. Ectopectoralis

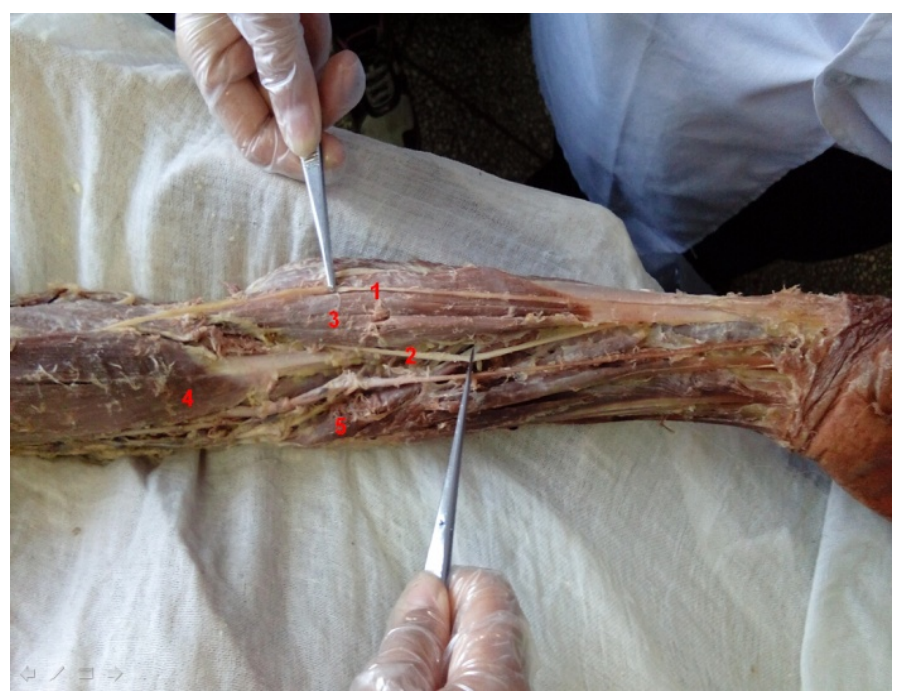

Figure 2. Variation of the lateral antebrachial cutaneous nerve and the superficial branch of the radial nerve dominating thumb-index web jointly (Middle forearm)

1. Lateral antebrachial cutaneous nerve, 2. Superficial radial nerve, 3. Brachioradialis, 4. Biceps brachii, 5. Pronation teres

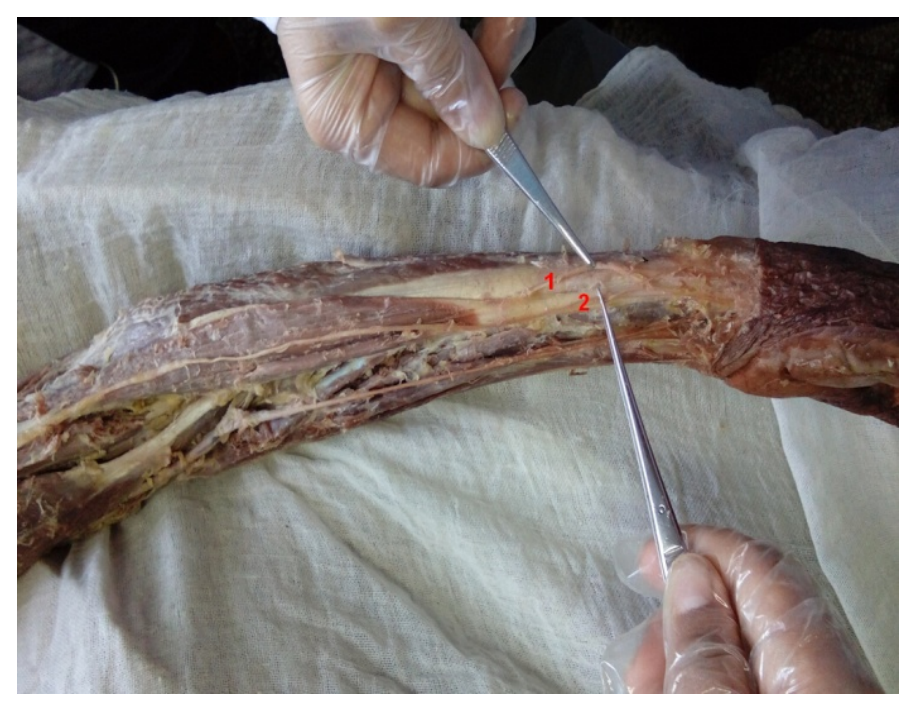

Figure 3. Variation of the lateral antebrachial cutaneous nerve and the superficial branch of the radial nerve dominating thumb-index web jointly (inferolateral forearm)

1. Superficial radial nerve, 2. Lateral antebrachial cutaneous nerve

consideration when the patient's radial nerve injured completely, for instance humeral midshaft fractures, but sensory function in the thumbindex web still remains [2]. In addition, when a patient is diagnosed with superficial radial nerve compression and the treatment effect of local block is not obvious, it might be due to the lateral antebrachial cutaneous nerve entrapment [3].

\section{Conclusion}

In conclusion, Detailed knowledge of the variation of median nerve and lateral antebrachial cutaneous nerve is clinical important in interpreting atypical clinical presentations or performing regional anaesthesia to the upper limb.

\section{References}

1. Atoni AD, CA Oyinbo (2017) Anatomic Variation of the Median Nerve Associated with an Anomalous Muscle of the Forearm. Folia Med (Plovdiv) 59: 106-109. [Crossref]

2. Davidovich ER, OJ Nascimento (2014) Superficial radial nerve-lateral antebrachial cutaneous nerve anatomic variation. Brain Behav 4: 70-74. [Crossref]

3. Muthulingam M, S Mahapatra (2016) Unusual Presentation of Radial Nerve Entrapment with Lateral Humeral Condyle Fracture - A Case Report. J Clin Diagn Res 10: RD04-RD06. [Crossref]

Copyright: (C2017 Jiang Z. This is an open-access article distributed under the terms of the Creative Commons Attribution License, which permits unrestricted use, distribution, and reproduction in any medium, provided the original author and source are credited. 